\title{
Expression of Spindle and Kinetochore-Associated Protein 1 Is Associated with Poor Prognosis in Papillary Thyroid Carcinoma
}

\author{
Chao Dong, Xiao-li Wang, and Bin-lin Ma \\ Department of Head and Neck Surgery, Tumor Hospital Affiliated to Xinjiang Medical University, Urumqi, Xinjiang 830011, China \\ Correspondence should be addressed to Bin-lin Ma; xjmabl@zoho.com
}

Received 6 February 2015; Accepted 16 April 2015

Academic Editor: Kishore Chaudhry

Copyright (C) 2015 Chao Dong et al. This is an open access article distributed under the Creative Commons Attribution License, which permits unrestricted use, distribution, and reproduction in any medium, provided the original work is properly cited.

\begin{abstract}
Aim. Spindle and kinetochore-associated protein 1 (SKA1) is one subtype of SKA, whose protein can make spindle microtubules attach steadily to the kinetochore in the middle of mitosis. At present, there are fewer researches on the relationship between SKA1 expression and tumor development. Methods. In this study, immunohistochemical analysis was used to determine the expression of SKA1 in papillary thyroid carcinoma (PTC) and adjacent tissues. We used quantitative real-time polymerase chain reaction (qRTPCR) and Western blot analysis to further verify the results. Results. We found that SKA1 expression was significantly higher in PTC tissues than normal adjacent tissues $(P<0.05)$. There existed a significant correlation among a higher SKAl expression, including lymphoid node $(P=0.005)$, clinical stage $(P=0.015)$, and extrathyroid invasion $(P=0.004)$. Survival analysis showed high SKA1 expression in PTC patients more likely to relapse after surgery. Conclusion. High SKA1 expression is predictive of poor prognosis of PTC, implying that SKA1 may be a promising new target for targeted therapies for PTC.
\end{abstract}

\section{Introduction}

Thyroid cancer is the most common malignant tumor of the endocrine system and the most common head and neck tumor. Each year, new cases of thyroid cancer account for $1-5 \%$ of all cancer cases [1]. Over the past 30 years, a rapid increase in the incidence of thyroid cancer has attracted widespread attention [2]. Thyroid cancer can be classified as papillary thyroid carcinoma (PTC), follicular thyroid carcinoma (FTC), medullary thyroid carcinoma (MTC), undifferentiated thyroid carcinoma (ATC), and others according to its pathological characteristics; PTC is the most common type, accounting for $79-94 \%$ of thyroid cancer cases [1]. With comprehensive surgery-focused antineoplastic therapy, the prognosis of most patients with PTC is good, but $20 \%$ these patients relapse after treatment and require reoperation [3]. Consequently, analyzing the biological characteristics of PTC and exploring new targets for therapy have been major clinical concerns.

It is well known that, during the cell cycle, the proper formation and depolymerization of the spindle play an essential role in maintaining normal mitosis of eukaryotic cells. However, chromosomal division abnormalities can cause genetic instability and ultimately induce PTC development [4]. Spindle and kinetochore-associated protein 1 (SKA1) is a subtype of SKA that causes spindle microtubules to attach firmly to the kinetochore in the middle of mitosis [5]. At present, few research studies have examined the relationship between SKA1 expression and tumor development. In our study, we use immunohistochemistry to observe SKA1 expression in PTC, and we conducted a quantitative analysis of SKA1 using quantitative real-time PCR and Western blotting with the aim of investigating the association between SKA1 and the prognosis of patients with PTC.

\section{Materials and Methods}

2.1. Patients and Samples. The subjects of this study were 123 patients with PTC who had undergone surgical intervention at the Department of Head and Neck Surgery of the Affiliated Tumor Hospital of Xinjiang Medical University between 2005 and 2009. All patients did not receive any therapy prior to surgery and were diagnosed with primary PTC based on the pathological findings. All 123 patients had complete clinical data, including age, sex, tumor size, lymph node status, clinical stage, and extrathyroidal invasion. Patients were 
selected for this study only if follow-up examinations and clinical data were available. The TNM stage was defined according to the 7th edition of the TNM classification of the International Union Against Cancer. The study was approved by the ethics committee of the Affiliated Tumor Hospital of Xinjiang Medical University. Written informed consent was obtained from each patient for the use of resected samples for research.

2.2. Immunohistochemistry. Biopsy tissue samples were sectioned into $4-\mu \mathrm{m}$ slices and embedded with paraffin after fixation with $10 \%$ formaldehyde. Immunohistochemistry with the SP method (rabbit anti-SKA1 antibody; 1:500; Sigma Chemical Co., St. Louis, MO, USA) was used for testing by applying PBS instead of primary antibodies as the negative control, according to the manufacturer's instructions. The results were determined by two independent pathologists using a double-blind method. Five high power fields (200x) were randomly selected, and in each field 200 tumor cells were recorded, totaling to 1000 cells. According to the staining intensity, the cells were assigned the following point values: 0 points for no staining; 1 point for pale yellow staining; 2 points for brown-yellow staining; and 3 points for dark brown staining. The proportion of positive cells was assigned a value between $0 \%$ and $100 \%$. The immunohistochemistry expression level was determined according to the product of the staining intensity and the proportion of positive cells. When the product was below $150 \%$, this was considered low expression of SKA1, and when the product was above $150 \%$, this was considered high expression of SKA1.

\subsection{RNA Extraction and Quantitative Real-Time PCR Assays.} The tissue samples were removed from liquid nitrogen, and RNA was extracted using Trizol reagent and a reverse transcription kit (Invitrogen; Carlsbad, CA, USA). The PCR primers used were SKA1-F: 5'-TGATGTGCCAGGAAGGTGAC-3', SKA1-R: $5^{\prime}$-CAAAGGATACAGATGAACAACAGC-3', GAPDH-F: $5^{\prime}$-GTGGACATCCGCAAAGAC$3^{\prime}$, and GAPDH-R: $5^{\prime}$-AAAGGGTGTAACGCAACTA-3'. All primers were synthesized by Shanghai Yingjun Biotechnology Company (Shanghai, China). The PCR reaction conditions were as follows: initial denaturation for $2 \mathrm{~min}$ at $94^{\circ} \mathrm{C}$, 35 cycles of denaturation for $30 \mathrm{~s}$, annealing for $30 \mathrm{~s}$ at $94^{\circ} \mathrm{C}$, and extension for $1 \mathrm{~min}$ at $72^{\circ} \mathrm{C}$, followed by $10 \mathrm{~min}$ at $72^{\circ} \mathrm{C}$. PCR reaction product $(3 \mu \mathrm{L})$ was used for $2 \%$ agarose gel electrophoresis, and the grayscale analysis was performed with Quantity One software (Bio-Rad; Hercules, CA, USA). The relative expression level of SKA1 mRNA was determined by the ratio of the grayscale value of SKA1 mRNA and that of internal reference GAPDH mRNA.

2.4. Western Blot Analysis. After protein extraction using total protein lysis buffer, the protein concentration was determined by the BCA method. In brief, $80 \mu \mathrm{g}$ protein per well was used for $8 \%$ SDS-PAGE, and after electrophoretic separation, the protein was wet-transferred to polyvinylidene fluoride; $5 \%$ nonfat milk powder solution was added, and after a $1 \mathrm{~h}$ incubation at room temperature, the transferred protein was incubated with the primary antibodies $(1: 500$ dilution SKA1 and GAPDH; Sigma Chemical Co., St. Louis, $\mathrm{MO}, \mathrm{USA})$ at $4^{\circ} \mathrm{C}$ overnight. Goat anti-rabbit IgG secondary antibody (GenScript Biotechnology Company, Nanjing, Jiangsu, China) marked with horseradish peroxidase and diluted at 1:1000 was added and incubated at room temperature for $1.5 \mathrm{~h}$. The membrane was washed three times in TBST. The relative level of SKA1 protein was determined by the grayscale value of various protein straps and the ratio of the grayscale value of SKA1 to GAPDH protein.

2.5. Statistical Analysis. The chi-squared test was used to analyze the associations between SKA1 expression and the clinicopathological features of PTC. SKA1 mRNA expression in PTC was compared with expression in normal adjacent tissues using the Mann-Whitney test (for 2 groups) or the Kruskal-Wallis test (for more than 2 groups). KaplanMeier analysis was used for survival analysis, lymph node recurrence-free survival (LNRFS), and distant recurrencefree survival (DRFS) calculations. LNRFS was defined as the time from the date of surgery to the date of lymph node relapse, and DRFS was defined as the time from the date of surgery to the date of distant recurrence $[6,7]$. Cox regression models were used for multiple factor analysis. All statistical tests were two-tailed, and statistical significance was assumed for $P<0.05$.

\section{Results}

3.1. Expression of SKA1 in PTC Tissues. Immunostaining results suggested a significantly higher SKA1 expression in PTC tissues compared with adjacent tissues. Among 123 tumor samples, 56 (45.5\%) showed high SKA1 expression, with only $37 \%$ of the samples from normal adjacent tissues (see Figure 1). To confirm these observations, we investigated SKA1 mRNA expression in 45 cases of PTC and normal adjacent tissues using qRT-PCR and Western blot analysis. The mRNA levels between PTC tissues and normal adjacent tissues were calculated with the comparative $\mathrm{Ct}$ method $\left(2^{-\Delta \Delta \mathrm{ct}}\right)$. The results indicated that SKA1 mRNA and SKA1 protein expression was significantly higher in PTC tissues than in normal adjacent tissues $(P<0.05$, Figures 2 and 3$)$.

3.2. Patient Characteristics. Table 1 shows the patients' clinical and immunohistochemical data. The mean age was $41.3 \pm$ 15.7 years (range 26-69 years) for 32 male patients and $44.2 \pm$ 14.5 years (range $24-73$ years) for 35 female patients. Sixtytwo patients $(50.4 \%)$ had lymph node metastasis. Eightyfour patients $(68.3 \%)$ had stage I-II disease based on the TNM staging system, and the remaining 39 patients $(31.7 \%)$ had stage III-IV disease. During the median follow-up time, which was 52 months (range 17-102 months), 21 of 123 patients $(17.1 \%)$ suffered lymph node recurrences, and 14 patients $(11.3 \%)$ had distant organ recurrences. As shown in Table 1, there was a significant association between higher SKA1 expression and lymph node involvement $(P=0.005)$, clinical stage $(P=0.015)$, and extrathyroidal invasion $(P=$ 0.004); however, no association was found between SKA1 

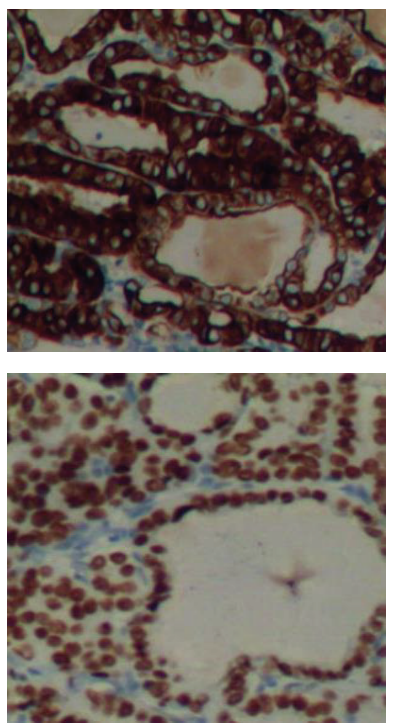

(a)


(b)
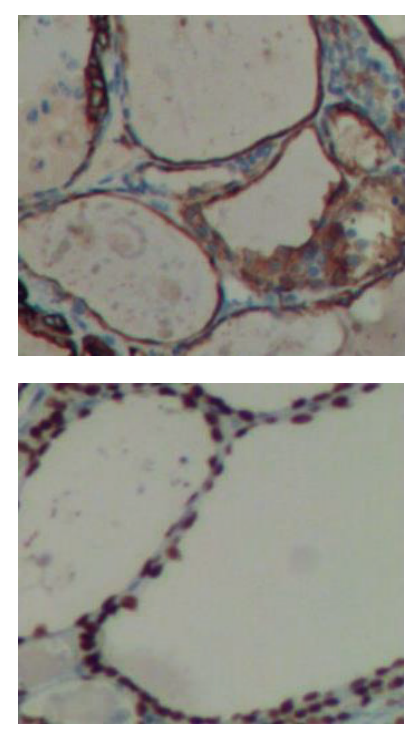

(c)

FIGURE 1: SKA1 expression by immunohistochemistry $(\mathrm{SP} \times 10)$. Left side is PTC tissue $(\mathrm{SP} \times 40)$; right side is normal adjacent tissue $(\mathrm{SP} \times$ $40)$.

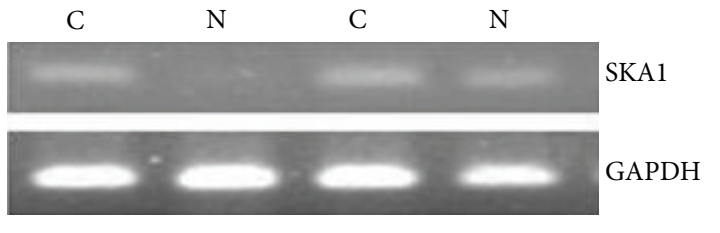

(a)

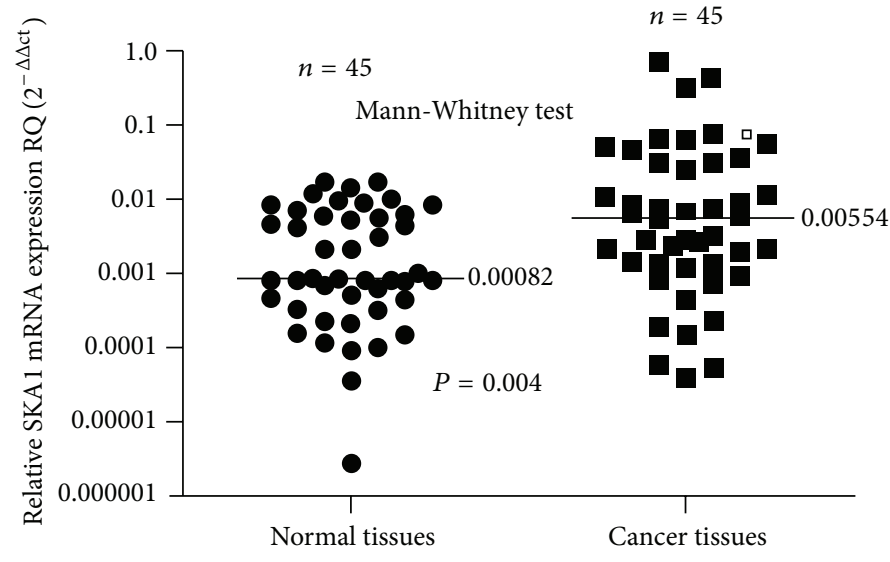

(b)

FIGURE 2: SKA1 mRNA was detected by qRT-PCR (N: normal tissues; C: cancer tissues).

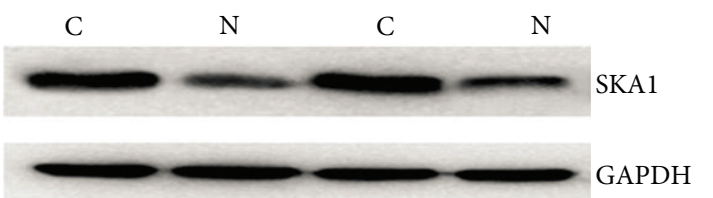

(a)

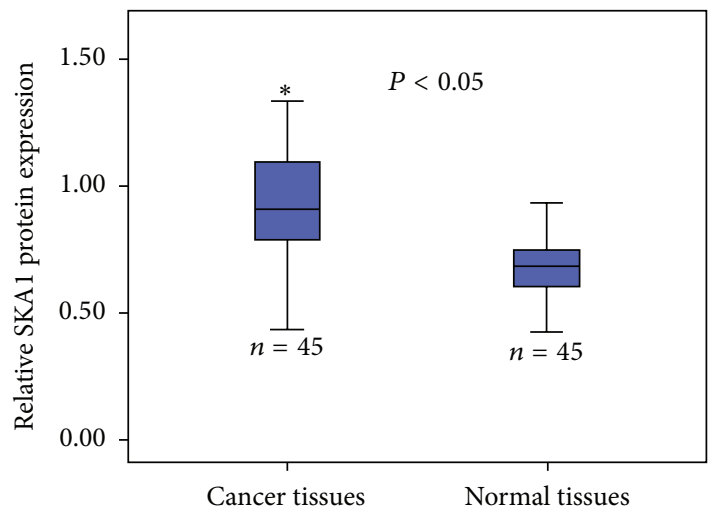

(b)

FIGURE 3: SKA1 protein was detected by Western blot analysis (N: normal tissues; C: cancer tissues). 
TABLE 1: Correlation of high SKA1 expression with clinicopathologic characteristics of PTC patients.

\begin{tabular}{|c|c|c|c|c|}
\hline \multirow{2}{*}{ Clinical features } & \multicolumn{2}{|c|}{ SKA1 expression } & \multirow{2}{*}{$\chi^{2}$} & \multirow{2}{*}{$P$ value } \\
\hline & Low $(n=67)$ & High $(n=56)$ & & \\
\hline \multicolumn{5}{|l|}{ Age } \\
\hline$<45$ years & 22 & 15 & \multirow{2}{*}{0.531} & \multirow{2}{*}{0.466} \\
\hline$\geq 45$ years & 45 & 41 & & \\
\hline \multicolumn{5}{|l|}{ Sex } \\
\hline Male & 32 & 23 & \multirow{2}{*}{0.552} & \multirow{2}{*}{0.457} \\
\hline Female & 35 & 33 & & \\
\hline \multicolumn{5}{|l|}{$\begin{array}{l}\text { Hashimoto's } \\
\text { thyroiditis }\end{array}$} \\
\hline Without & 64 & 51 & \multirow{2}{*}{0.994} & \multirow{2}{*}{0.467} \\
\hline With & 3 & 5 & & \\
\hline \multicolumn{5}{|l|}{ Tumor size } \\
\hline$<2 \mathrm{~cm}$ & 42 & 26 & \multirow{2}{*}{1.008} & \multirow{2}{*}{0.3799} \\
\hline$\geq 2 \mathrm{~cm}$ & 25 & 30 & & \\
\hline \multicolumn{5}{|l|}{ Multifocality } \\
\hline Without & 43 & 34 & \multirow{2}{*}{0.156} & \multirow{2}{*}{0.712} \\
\hline With & 24 & 22 & & \\
\hline \multicolumn{5}{|l|}{ Differentiation } \\
\hline Well & 19 & 17 & \multirow{2}{*}{0.059} & \multirow{2}{*}{0.808} \\
\hline Poor & 48 & 39 & & \\
\hline \multicolumn{5}{|l|}{ Lymphoid node } \\
\hline Without & 41 & 20 & \multirow{2}{*}{7.922} & \multirow{2}{*}{0.005} \\
\hline With & 26 & 36 & & \\
\hline \multicolumn{5}{|l|}{ Clinical stage } \\
\hline I-II & 52 & 32 & \multirow[t]{2}{*}{5.902} & \multirow[t]{2}{*}{0.015} \\
\hline III-IV & 15 & 24 & & \\
\hline \multicolumn{5}{|l|}{$\begin{array}{l}\text { Extrathyroid } \\
\text { invasion }\end{array}$} \\
\hline Without & 46 & 24 & \multirow{2}{*}{8.280} & \multirow{2}{*}{0.004} \\
\hline With & 21 & 32 & & \\
\hline
\end{tabular}

expression and other clinicopathological features. According to the data, SKA1 expression in tumors might be useful for identifying the degree of PTC.

3.3. Expression of SKA1 and Prognosis of PTC Patients. We used the Kaplan-Meier method to analyze the relationship between SKA1 expression levels and the prognosis of patients with PTC. The results showed that high SKA1 expression was associated with LNRFS $(P<0.001)$ and DRFS $(P<$ 0.001 ) (Table 2, Figures 4 and 5). We performed multivariate analysis using the Cox regression model. The results of multivariate analysis for the mentioned parameters showed that high expression of SKA1 was an independent indicator of LNRFS and DRFS (Table 3).
TABLE 2: LNRFS and DRFS of PTC patients with high and low SKA1 expressions.

\begin{tabular}{lccc}
\hline & $N$ & $\begin{array}{c}\text { Survival time (months) } \\
\mathrm{M} \pm \mathrm{SE}\end{array}$ & $P$ value \\
& & & \\
LNRFS & & $84.31 \pm 2.34$ & $<0.001$ \\
SAK1 low expression & 67 & $51.96 \pm 3.38$ & \\
SAK1 high expression & 56 & & \\
DRFS & & $87.14 \pm 2.06$ & $<0.001$ \\
SAK1 low expression & 67 & $56.25 \pm 3.23$ & \\
SAK1 high expression & 56 &
\end{tabular}

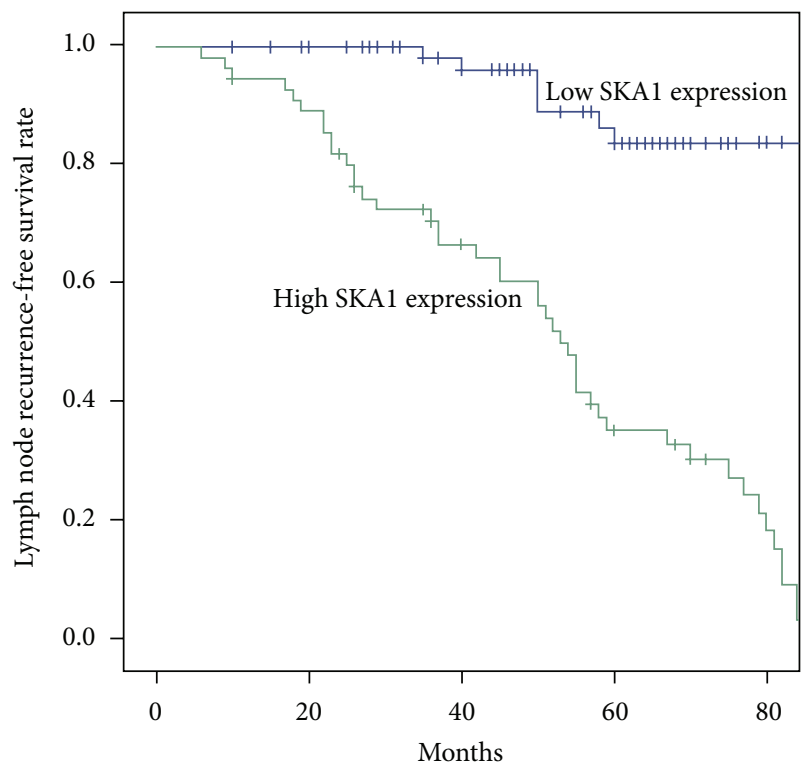

FIGURE 4: SKA1 expression and LNRFS.

\section{Discussion}

An equal distribution of chromosomes depends on the precise regulation of mitosis during each cell cycle, and the correct formation and depolymerization of the spindle are important for the regulation process [8]. Abnormalities of mitosis can lead to aneuploidy and genomic instability, ultimately resulting in cell death or tumorigenesis [9, 10]. The spindle, a temporary organelle directly related to cell division and chromosome movement, is composed of microtubules and conjugated proteins, and the kinetochore, a large protein complex, is an important link between mitotic chromosomes and spindle microtubules [11]. Based on one significant assumption, spindle orientation defects can lead to cell number increase by suppressing the asymmetric division of stem cells while promoting their symmetric proliferation $[12,13]$. Furthermore, tissue architecture disorganization, a typical feature of malignant transformation, might occur due to defective spindle orientation $[12,14]$.

The SKA1 gene is located on chromosome 18q21.1, contains 255 amino acids, and is approximately $30 \mathrm{kDa}$ in size [15]. SKA1 is a necessary component for a stable kinetochore and microtubule binding, and it might $=$ promote 
TABle 3: Multivariate analysis of the prognosis of PTC.

\begin{tabular}{|c|c|c|c|c|}
\hline Variable & $\begin{array}{c}\text { LNRFS } \\
\text { HR for death }(95 \% \mathrm{CI})\end{array}$ & $P$ value & $\begin{array}{c}\text { DRFS } \\
\text { HR for death }(95 \% \mathrm{CI})\end{array}$ & $P$ value \\
\hline Lymphoid node & $1.899(1.054-3.422)$ & 0.033 & $2.089(1.144-3.817)$ & 0.017 \\
\hline Clinical stage & $2.827(1.485-5.384)$ & 0.002 & $3.026(1.573-5.822)$ & 0.001 \\
\hline Extrathyroid invasion & $2.275(1.197-4.324)$ & 0.012 & $2.490(1.305-4.751)$ & 0.006 \\
\hline SKA1 high expression & $4.862(2.071-11.412)$ & $<0.001$ & $4.907(2.099-11.475)$ & $<0.001$ \\
\hline
\end{tabular}

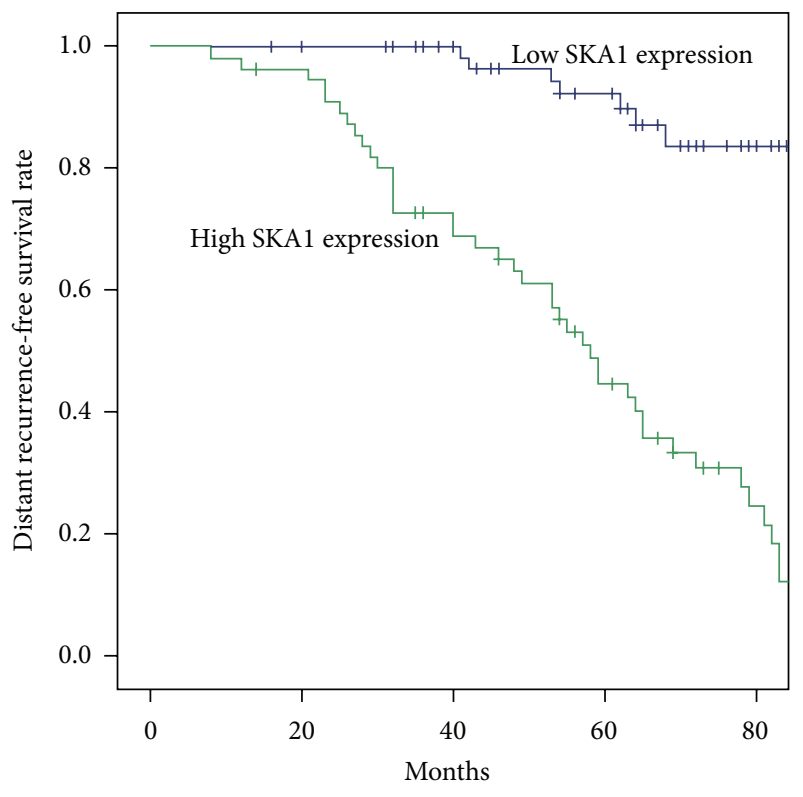

FIGURE 5: SKA1 expression and DRFS.

the combination of kinetochore and tubulin and regulate the depolymerization of tubulin and the synchronization of chromosomal migration and movement towards the 2 poles [16]. The SKA1 protein might be expressed not only in the spindle but also in the kinetochore [17]. Its carboxyl terminus contains a conserved microtubule-binding domain with multiple phosphorylation sites that can be phosphorylated by Aurora B kinase to further regulate the combination of SKA1 and microtubules [18]. Formation of the SKA complex helps to ensure the correct positioning of the spindle and kinetochore to promote the transition from middle to late mitosis.

Li et al. [19] found that SKA1 was overexpressed in human prostatic intraepithelial neoplasia by immunohistochemistry and quantitative RT-PCR. They also carried out in vitro experiments, which showed that prostate-specific upregulation of SKA1 in a transgenic mouse model resulted in spontaneous tumorigenesis [19]. In addition, SKA1 is a critical factor that plays an essential role in the regulation of hepatocellular carcinoma cell proliferation and apoptosis; research showed that knockdown of SKA1 inhibited hepatocellular carcinoma cell proliferation by inducing cell cycle arrest in the G0/G1 phase [20]. The few studies on this indicate that abnormal SKA1 expression might be closely associated with the development of other solid tumors, such as gastric cancer, oral adenosquamous carcinoma, and neuronal glioblastoma $[5,21,22]$. In this study, we found that SKA1 expression was associated with the clinicopathological features and prognosis of patients with PTC. Our results suggest that a high expression of SKA1 might be associated with lymph node status, clinical stage, and extrathyroidal invasion, further implying an involvement of SKA1 in the incidence and development of PTC. In addition, we demonstrated an association between high SKA1 expression and the prognosis of patients with PTC using Kaplan-Meier survival curves. Our findings indicate that a high expression of SKA1 indicates worse LNRFS and DRFS. At the same time, our multivariate analysis using Cox proportional hazards regression model showed that high expression of SKA1 was related to PTC patient prognosis. Based on these results, SKA1 might be an important prognostic indicator for PTC.

To the best of our knowledge, this is the first report on the relationship between SKA1 and prognosis in patients with PTC. High SKA1 expression is predictive of poor prognosis in PTC, implying that SKA1 might be a promising new target for targeted therapies for PTC. However, to determine the mechanism by which SKA1 upregulated expression promotes PTC generation and development and whether SKA1 also has abnormal expression in other solid tumors, further study is needed.

\section{Disclaimer}

The funders had no role in study design, data collection and analysis, decision to publish, or preparation of the paper.

\section{Conflict of Interests}

The authors have declared that no competing interests exist.

\section{Acknowledgment}

This study was funded by the Natural Science Foundation of the Xinjiang Uyghur Autonomous Region (Grant no. 2011211A036) and Research and Innovation Foundation of Xinjiang Medical University (Grant no. XJC201270).

\section{References}

[1] J. A. Sipos and E. L. Mazzaferri, "Thyroid cancer epidemiology and prognostic variables," Clinical Oncology, vol. 22, no. 6, pp. 395-404, 2010. 
[2] V. Zivaljevic, N. Slijepcevic, S. Sipetic et al., "Risk factors for well-differentiated thyroid cancer in men," Tumori, vol. 99, no. 4, pp. 458-462, 2013.

[3] S. Young, A. Harari, S. Smooke-Praw, P. H. G. Ituarte, and M. W. Yeh, "Effect of reoperation on outcomes in papillary thyroid cancer," Surgery, vol. 154, no. 6, pp. 1354-1362, 2013.

[4] S. Cantara, M. Pisu, D. V. Frau et al., "Telomere abnormalities and chromosome fragility in patients affected by familial papillary thyroid cancer," Journal of Clinical Endocrinology and Metabolism, vol. 97, no. 7, pp. E1327-E1331, 2012.

[5] B. Zhang, K. Y. Li, H. Y. Chen et al., "Spindle and kinetochore associated complex subunit 1 regulates the proliferation of oral adenosquamous carcinoma CAL-27 cells in vitro," Cancer Cell International, vol. 13, no. 1, article 83, 2013.

[6] Y. H. Park, S. J. Lee, E. Y. Cho et al., "Clinical relevance of TNM staging system according to breast cancer subtypes," Annals of Oncology, vol. 22, no. 7, pp. 1554-1560, 2011.

[7] NSCLC Meta-analysis Collaborative Group, "Preoperative chemotherapy for non-small-cell lung cancer: a systematic review and meta-analysis of individual participant data," The Lancet, vol. 383, no. 9928, pp. 1561-1571, 2014.

[8] H. S. Sardar and S. P. Gilbert, "Microtubule capture by mitotic kinesin centromere protein E (CENP-E)," Journal of Biological Chemistry, vol. 287, no. 30, pp. 24894-24904, 2012.

[9] L. Kabeche and D. A. Compton, "Cyclin A regulates kinetochore microtubules to promote faithful chromosome segregation," Nature, vol. 502, no. 7469, pp. 110-113, 2013.

[10] K. Crasta, N. J. Ganem, R. Dagher et al., "DNA breaks and chromosome pulverization from errors in mitosis," Nature, vol. 482, no. 7383, pp. 53-58, 2012.

[11] H. Wang, I. Brust-Mascher, and J. M. Scholey, "Sliding filaments and mitotic spindle organization," Nature Cell Biology, vol. 16, no. 8, pp. 737-739, 2014.

[12] A. Noatynska, M. Gotta, and P. Meraldi, "Mitotic spindle (DIS)orientation and DISease: cause or consequence?" Journal of Cell Biology, vol. 199, no. 7, pp. 1025-1035, 2012.

[13] S. J. Morrison and J. Kimble, "Asymmetric and symmetric stemcell divisions in development and cancer," Nature, vol. 441, no. 7097, pp. 1068-1074, 2006.

[14] S. S. McAllister and R. A. Weinberg, "Tumor-host interactions: a far-reaching relationship," Journal of Clinical Oncology, vol. 28, no. 26, pp. 4022-4028, 2010.

[15] M. A. Abad, B. Medina, A. Santamaria et al., "Structural basis for microtubule recognition by the human kinetochore Ska complex," Nature Communications, vol. 5, article 2964, 2014.

[16] D. A. Skoufias, C. Mollinari, F. B. Lacroix, and R. L. Margolis, "Human survivin is a kinetochore-associated passenger protein," Journal of Cell Biology, vol. 151, no. 7, pp. 1575-1582, 2000.

[17] G. J. Guimaraes and J. G. Deluca, "Connecting with Ska, a key complex at the kinetochore-microtubule interface," $E M B O$ Journal, vol. 28, no. 10, pp. 1375-1377, 2009.

[18] A. Boeszoermenyi, J. C. Schmidt, I. M. Cheeseman, M. Oberer, G. Wagner, and H. Arthanari, "Resonance assignments of the microtubule-binding domain of the C. elegans spindle and kinetochore-associated protein 1," Biomolecular NMR Assignments, vol. 8, no. 2, pp. 275-278, 2014.

[19] J. Li, J. W. Xuan, V. Khatamianfar et al., "SKA1 over-expression promotes centriole over-duplication, centrosome amplification and prostate tumourigenesis," The Journal of Pathology, vol. 234, no. 2, pp. 178-189, 2014.
[20] X. Qin, B. Yuan, X. Xu, H. Huang, and Y. Liu, "Effects of short interfering RNA-mediated gene silencing of SKA1 on proliferation of hepatocellular carcinoma cells," Scandinavian Journal of Gastroenterology, vol. 48, no. 11, pp. 1324-1332, 2013.

[21] W. Sun, L. Yao, B. Jiang, L. Guo, and Q. Wang, "Spindle and kinetochore-associated protein 1 is overexpressed in gastric cancer and modulates cell growth," Molecular and Cellular Biochemistry, vol. 391, no. 1-2, pp. 167-174, 2014.

[22] X. Shi, X. Chen, H. Peng et al., "Lentivirus-mediated silencing of spindle and kinetochore-associated protein 1 inhibits the proliferation and invasion of neuronal glioblastoma cells," Molecular Medicine Reports, vol. 11, no. 5, pp. 3533-3538, 2015. 


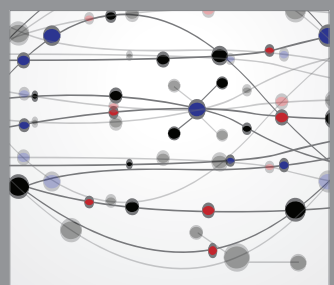

The Scientific World Journal


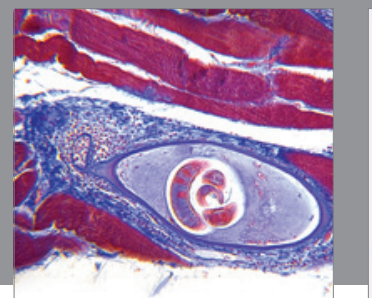

Gastroenterology

Research and Practice
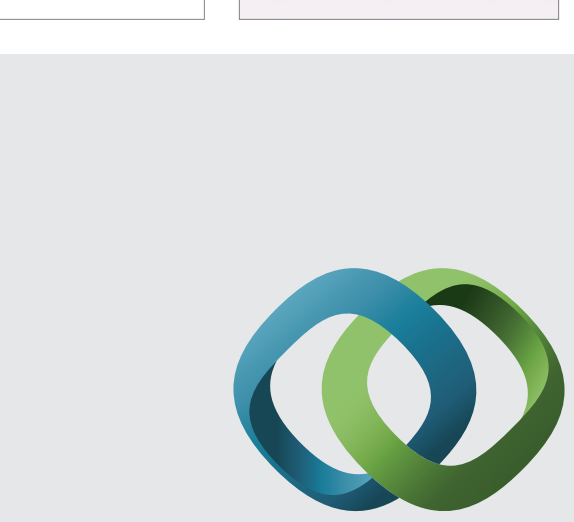

\section{Hindawi}

Submit your manuscripts at

http://www.hindawi.com
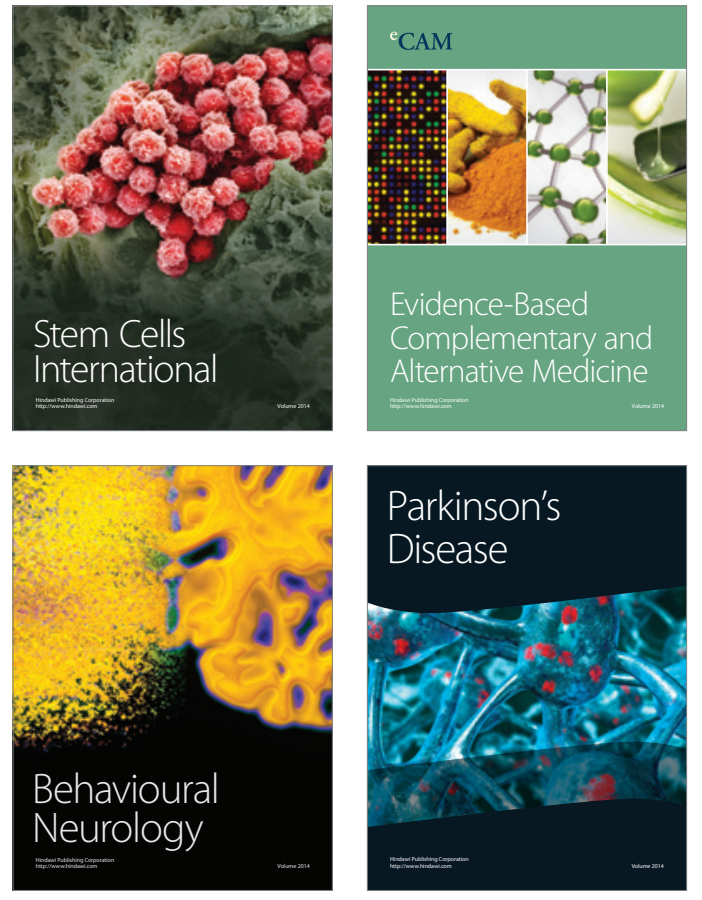
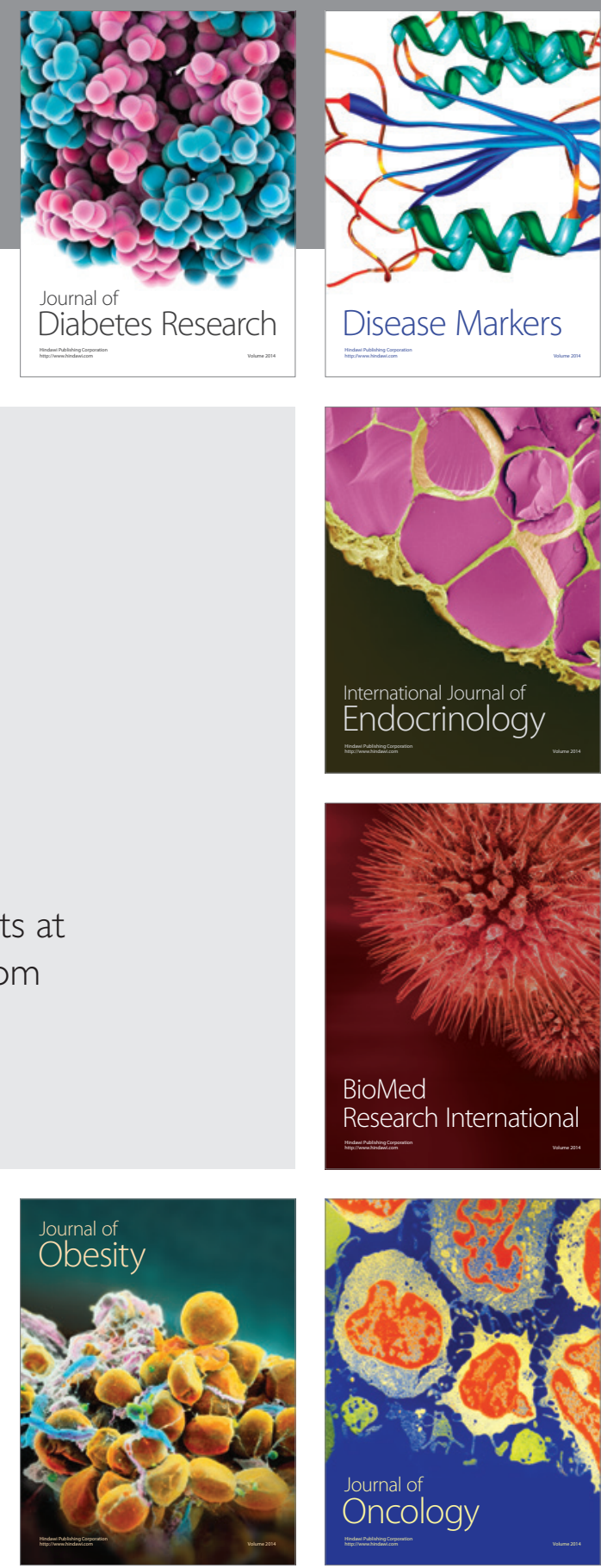

Disease Markers
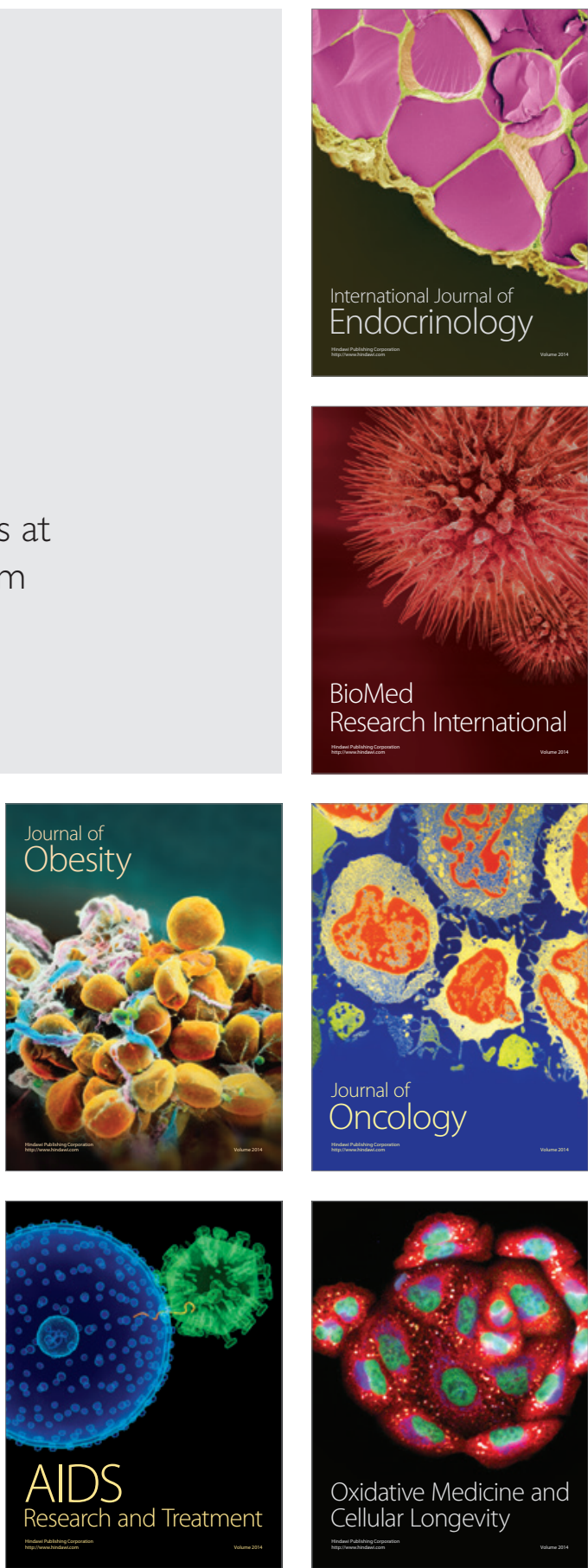